\title{
Design on English Grammar Comprehensive Query System based on SSH Framework
}

\author{
Haiyan Feng \\ Department of Foreign Languages, Liaoning Institute of Science and Engineering, Jinzhou, 121013, \\ China \\ Fenghaiyan06214@163.com
}

Keywords: SSH framework; English grammar; comprehensive query; system design

\begin{abstract}
Any language has its inherent rules and laws, the rules of the grammar is a set of words, is the tie that vocabulary and sentences are important to the language conditions, from the essence of language, the practical characteristics of English teaching, the cognitive perspective, English grammar is the most important part of English teaching. Comprehensive query system based on SSH framework design of English grammar provide convenient query syntax for students and teachers. System will be grammar content stored in the database can query syntax items, also can inquire details. Grammar can be divided into parts of speech, syntactic system, clauses, voice, tense, subjunctive mood comprehensive grammar and usage analysis such as the eight classes, the user can also be queried according to category.
\end{abstract}

\section{Introduction}

Grammar is a branch of linguistics, including two meanings: one refers to the grammatical structure rule itself, namely grammar; two is refers to the grammar, the science of exploration and descriptive grammar structure, grammar is grammar scholar of objective existence and instructions.

Any language has its inherent grammar rules and laws, rules of grammar is a set of words, is the glue of vocabulary and sentence, is extremely important conditions of language. Grammar can be divided into two parts of the lexical and syntactic, lexical including all kinds of word form and its changes; Syntax of main teaching sentence types and type, parts of the sentence and the law of the phrasing. English grammar is for English language study, systematically summarized out a series of language rules. In terms of the nature of language, English teaching and the characteristic and the cognitive point of view, English grammar is an important part of English teaching. There are a number of problems with the current college English grammar teaching. Teachers mainly adopt methods such as grammar rules and practice problems. The content of the teaching is given priority to with textbook knowledge, but the textbook knowledge theory is very strong, students accept more difficult. From the point of curriculum arrangement, teaching contents and teaching hours too little too much, in order to meet the needs of improving test scores, but also increase the exam-oriented teaching content, brings great pressure to grammar teaching. Single way of teaching and learning, can't stimulate students interest, prone to resistance, English grammar teaching efficiency is low, has become the bottleneck of teaching and students' learning difficulties.

Software framework is a set of related abstract model, used to guide design aspects of large software systems. Software framework is a sketch of a system, which is based on certain design principles, the parts that make up the system from different angles for collocation and arrangement, form a system of multiple structure and component framework, including the system of individual components, components of the externally visible properties and the relationship between components. Software framework to complete the transformation from business oriented to face technology, with shorter development cycle, improve software quality, reduce costs, easy to modify and maintain, etc. J2EE is the server-side programming technology based on Java language and J2EE platform is a multi-layer distributed application model, retain the existing IT resources, with the development of high efficiency, support heterogeneous environment, excellent and stable availability scalability. J2EE platform application logic is encapsulated into components according 
to the function, the client layer components, Web components, business logic layer and business layer components, client through the browser to realize the database interaction.

SSH framework are Struts framework, Spring framework and Hibernate framework of integrated framework, is a mainstream of the J2EE lightweight framework, based on the presentation layer, business logic layer and data layer between the dependence of consolidation, implements the loose coupling between the layers. SSH framework structure clear, be helpful for developers to reduce the workload. English grammar comprehensive query system based on SSH framework design and development, to provide teachers and students English grammar query service system, for the convenience of grammar learning and improve English teaching level of service.

\section{SSH Framework Structure}

SSH framework structure as shown in Fig. 1.

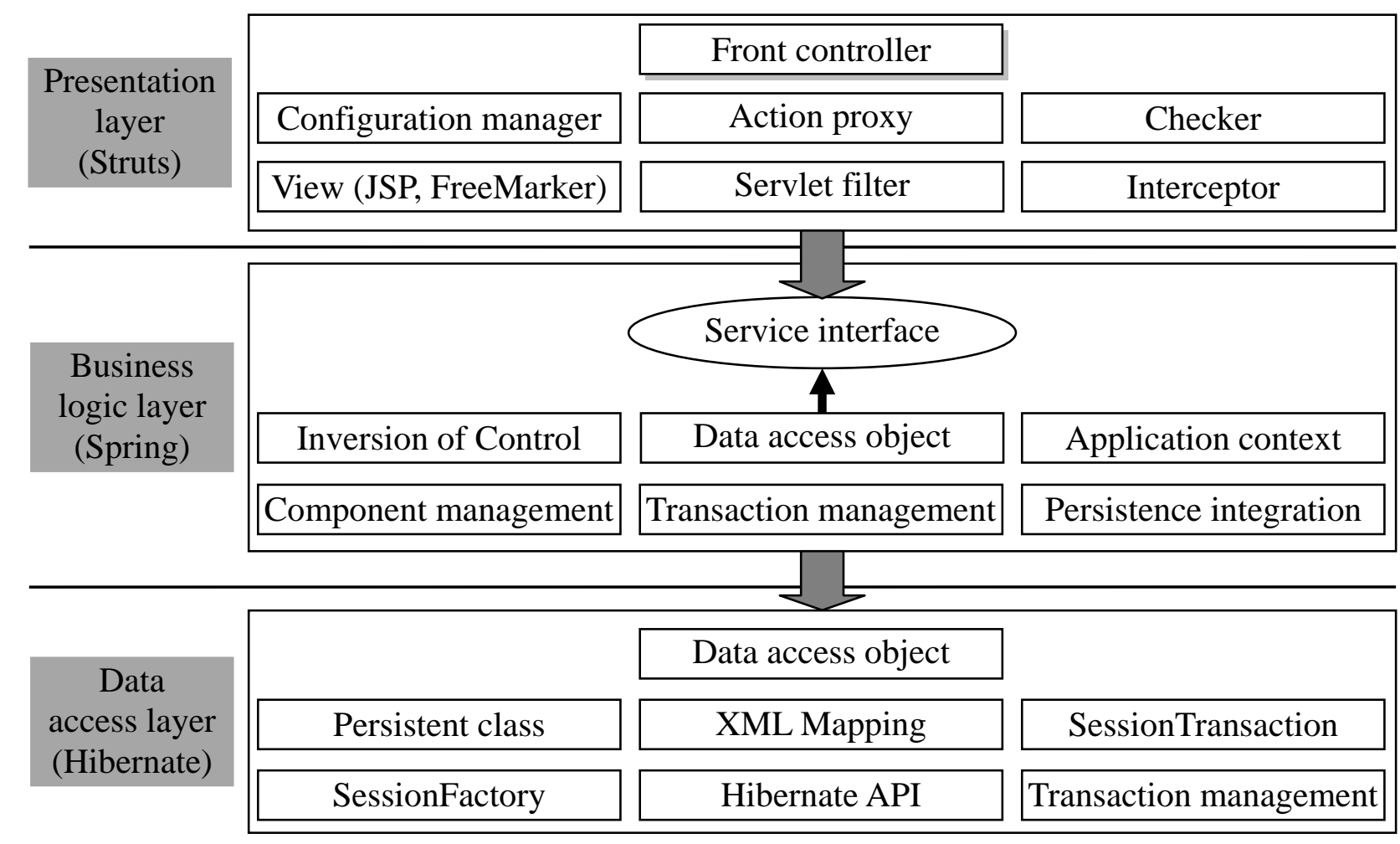

Fig. 1. SSH Framework Structure

Struts framework USES the Struts 2 core component is the Action class, encapsulates the client's request and invokes the business layer of the Service class, through the web. The XML file configuration filter, by Struts. The XML file configuration client page request. A Web request in the Struts2 framework, the process is: the client after initialization point to the Servlet request; Request after filter called and asked to decide whether to need to call an Action; If you need to call you using ActionProxy framework via the Configuration Manager profile information, find the need to call the Action class, and then create the ActionInvocation instance; Last call Action, after the Action has been completed according to the struts. Find corresponding XML to return the result.

Spring framework is a lightweight framework a multi-layer system structure based on AOP and the IOC. AOP technology is mainly used for separating the business logic and system services enhance cohesion; IOC technology is used to remove excessive coupling between the programs and simplify the development. The Spring framework is mainly responsible for organizing the business logic module to work together, the program contains the business logic and data access objects, service and transaction management organized by the configuration file. Spring's core is to support the reusable business and data access object, based on time delay into the assembly code, to increase the extensibility and flexibility of the system, implements the plug-in programming; AOP thought reduce code duplication rate, promote the effectiveness of the solution. 
Hibernate is an ORM (Object Relational Mapping) framework, the role is to simplify the application of data persistence layer programming, don't need to spend a lot of time to write SQL programmers and JDBC code. Framework mainly includes the persistent objects, attributes, and map files, records in the persistent object is used to map database, a properties file used for programming of data persistence layer, the mapping file is an XML file, named by the form of *.hbm.xml1, used to represent a mapping relation. The corresponding reflection mechanism to achieve transparency through the Java; HQL statement is simplified, and improve the development efficiency; Can directly take packaging strategy, do not need to write your own code but by mapping the object as a transmission medium to transfer.

\section{System Requirement Analysis}

Requirement analysis is an important work of the software life cycle, and decisive. Only through the demand analysis, the software function and performance of the overall concept, described as a specific software requirements specification, so as to lay the foundation of software development. Demand analysis is important, because have the function of the decision-making, directivity, strategic, has a pivotal position in the software development process. Requirement analysis is an effective way to get users demand, demand analysis is the key factor that decides the success of the project, the requirements analysis is the bridge of system analysis and software design, requirement analysis is an important stage of software quality control. Data flow diagrams demand analysis is usually used to describe the logical model of the system. According to the hierarchical data flow diagrams are divided into the top, middle data flow graph and data flow diagram the underlying data flow diagram. In this paper, the design of the system top level data flow diagram, as shown in Fig. 2.

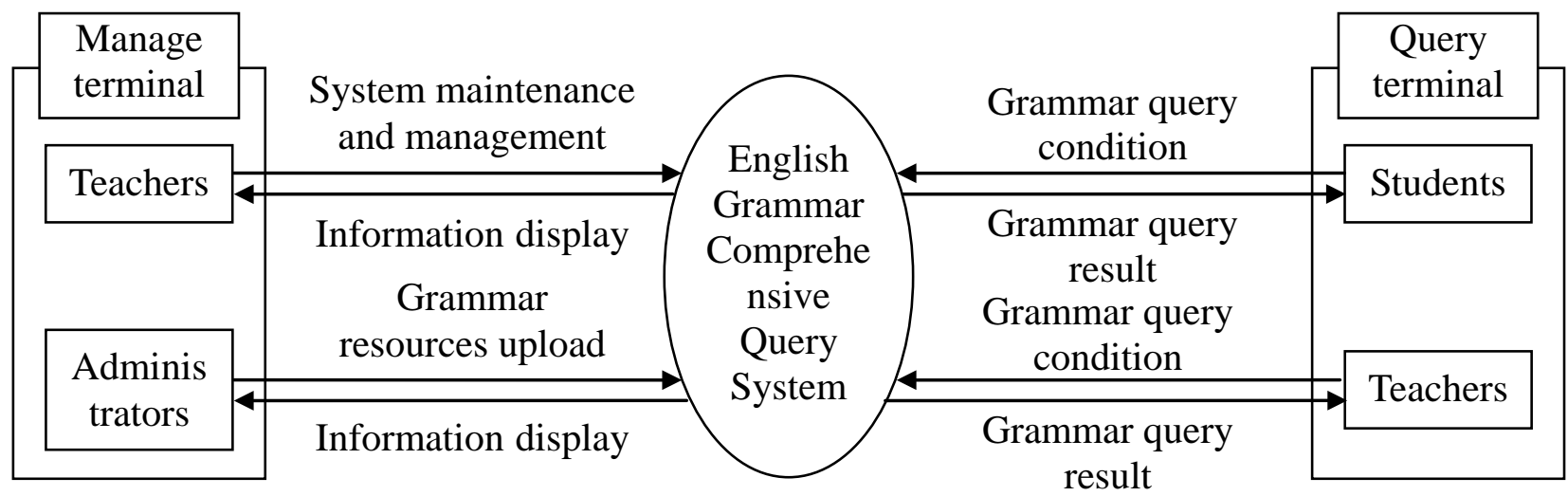

Fig. 2. System top level data flow diagram on English grammar comprehensive query system

According to the Fig. 2, the system consists of "two types of terminal" and "three kinds of users". There are two types of terminal respectively management terminal and the client terminal. The three types of users are administrators, teachers and students. Terminal is the main function of the system management and resource management main function is to query syntax, grammar, the client terminal. Administrator user functions include configuration management, data maintenance, user management and resource management, and so on, can also release related notice or usage, and so on, end users, teacher management core function is uploaded to the database server, syntax and grammar resources to maintain. Students query using user's core function is grammar, according to their own need to input the query conditions, obtain the corresponding results can also be released for grammar or information resource evaluation; Client terminal users, teachers and students, also have the function of syntax queries, in the process of teaching or learning, can also according to their own need to query the corresponding grammar knowledge.

\section{System Function Design}

Function refers to the object can meet the demand of a certain kind of attributes. Software function 
is a software should have the efficiency and effect, software goal through software functions to express and implementation, software function and software is presented to the user directly effect. User through software provides functions to understand, use and evaluation system, through the use of the software functions to complete the operation. Profile design also calls the function design, main is clear the specific function of the software development, provides reference for the follow-up of software development. Specific task is a complex system module partition according to function, to establish the hierarchical structure of the module and call relationship, determine the interface between the module and human-computer interface, etc. The functional design of commonly used method is Modularity. Modularity is an important kind of design thought, a complex system is decomposed into some smaller and more simple, more easy to build and modify the part. Many English grammar comprehensive query system function, the main features include four items, as shown in Fig. 3.

\begin{tabular}{|c|c|}
\multicolumn{2}{c|}{ English Grammar Comprehensive Query System } \\
\hline Grammar classification management & Grammar comprehensive query \\
\hline Grammar resources upload & User rights management \\
\hline
\end{tabular}

Fig. 3. Function on English grammar comprehensive query system

Core functions of the system include the following four:

(1) Grammar classification management. English grammar is more, only good design classification, students can easily query according to their own needs. Grammar can be divided into two parts of lexical and syntactic, lexical include various categories of word form and its change, the syntactic teaching of sentence types and main types. Usually the classification of grammar is as shown in Fig. 4. In Fig. 4 said tree structure, cannot be stored in a relational database. Only is a two-dimensional table form of norms, in accordance with relational database storage practices. Therefore, in order to facilitate the storage, the English grammar classified into eight categories, respectively is the part of speech, syntactic, clauses, voice, tense, subjunctive mood, grammar and usage. Those not belong to more than eight classes of grammar as the "other" category.

(2) Grammatical resources to upload. Grammar resources uploaded to the server only, ability is used by the query. Therefore, grammar resources upload is an important function of the system, upload is usually done by teachers' users, students users can also be good grammar but require teachers review to user queries. Data upload is general function of the software, and more simple, to design the user upload grammar resources, need to fill in the basic information of the grammatical resources, including the classification number, name of classification, source of grammar, syntax, grammar, the author, grammar title and source address content, etc. If the content of the grammatical resources is more, you can upload in the form of attachment. If the content is less, you can be directly copied to the grammar.

(3) Comprehensive query syntax. It is the core function of this system for teachers and students users, including grammar items query and query two interfaces in detail. Grammar items query, query input conditions first and then satisfied condition of grammar items. Query conditions including the classification number, name of the classification, source of syntax, grammar, the author, important grade, descendants on grammar descendants on the name, number and title, etc. Can enter one of the conditions or more, is "or" relationship between multiple conditions. The query result to the screen in the form of a list. Click on the title of a grammar items links, syntax details into the interface, the main display syntax details and examples, the user through the interface for grammar learning.

(4) User permission management. This system including administrators, teachers and students and so on three types of users, for the safety of system, each type of users with different permissions. Will be assigned to different users according to their need of function in the corresponding group (group is a collection of permissions, equivalent to the role of the database management system), different groups have different permissions. System maintenance personnel planning first user 
group, the users are assigned to the corresponding group, assign permissions for group. System login first determines the user's group, the group permissions assigned to users, will not have the function of automatic block, so as to ensure the confidentiality, integrity and accuracy of data.

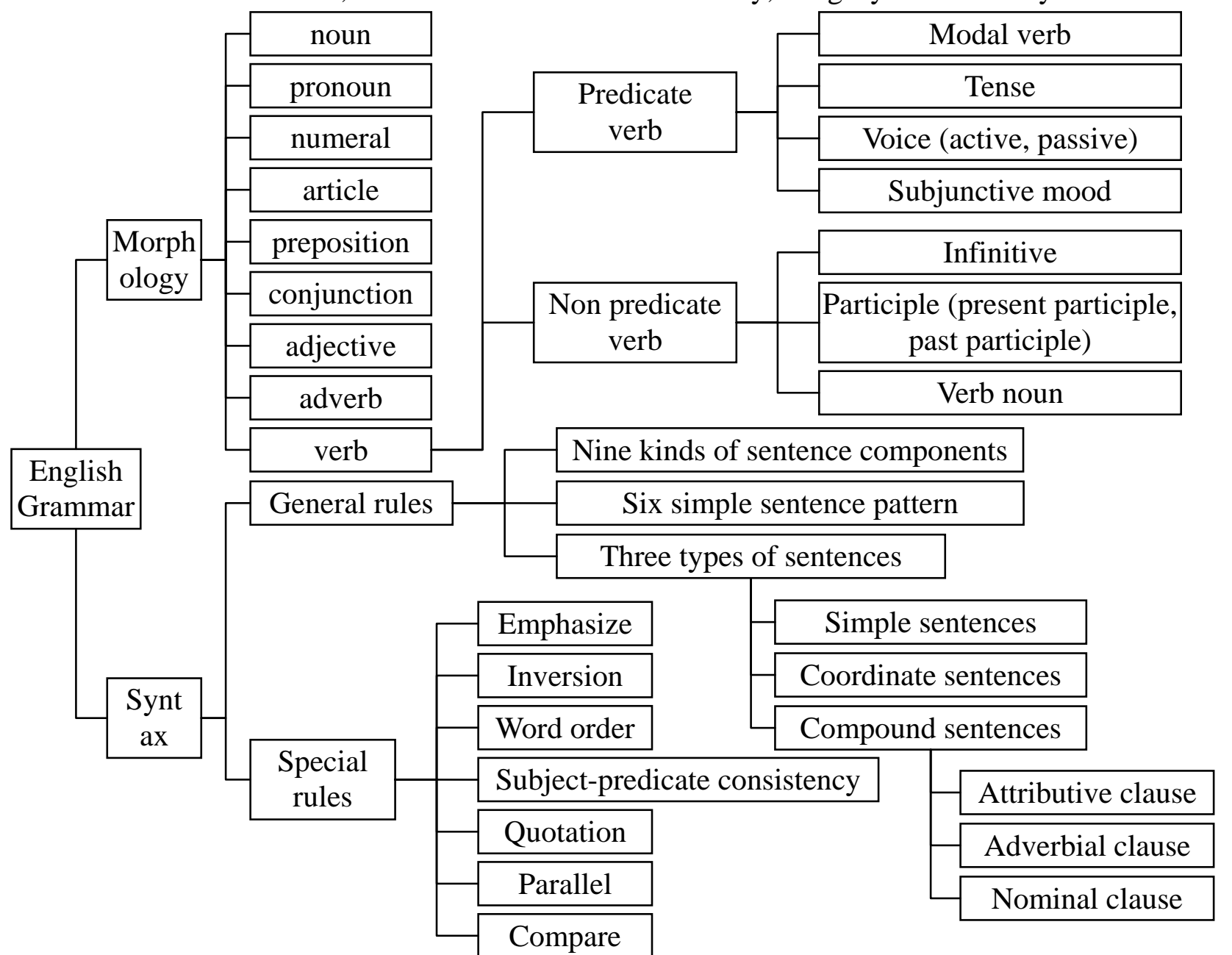

Fig. 4. Classification of English grammar

\section{References}

[1] L. Y. Wang, H. Wu, L. Y. Wang, "Design and Realization of English Grammar Mobile Learning System Based on Android," Computer and Modernization, vol. 29, no. 8, pp. 232-235, 2013.

[2] X. F. Pang, "On the reform of College English grammar teaching model," Journal of Higher Education, vol. 3, no. 2, pp. 136-137, 2012.

[3] J. Lu, "College English grammar teaching guided by constructivism teaching theory," Journal of Educational Institute of Jilin Province, vol. 32, no. 5, pp. 56-58, 2016.

[4] G. C. Wang, "The Design and Implementation of AOP Examination System Based on SSH Framework," Master's Degree of Guizhou Normal University, 2014.

[5] Y. S. Zhang, X. C. Cheng. The Design and Implementation of Hibernate-based Persistence Layer, Computer Technology and Development, vol. 24, no. 12, pp. 102-103, 2014.

[6] L. X. Yang, Y. Yao, "The Design of SSH-based Multilayer Architecture," Yinshan Academic Journal, vol. 26, no. 4, pp. 15-17, 2012. 